\title{
5G, and technologies that will make it happen
}

\section{Rahul Darmwal}

\author{
Sr. Consultant, Ericsson, Mumbai, India
}

Abstract: The next generation of wireless networks are already being deployed in advanced markets. This $5^{\text {th }}$ generation wireless technology promises to improve network performance by factors of 10-1000's, thus providing unlimited potential in consumer and enterprise segments. In this paper, I will try to explain how $5 G$ aims to reach its planned capabilities through use of new technologies such as mm Wave, Network Virtualization, Edge computing and Network Slicing.

Keywords: 5G, mmWave, cloud, network virtualization, network slice, edge computing

\section{Introduction}

Mobile telephony has come a long way in last few decades. Starting from circuit switched basic voice calling in 1980's, it has now reached a state where voice is just an application being transported on all IP packet switched networks.

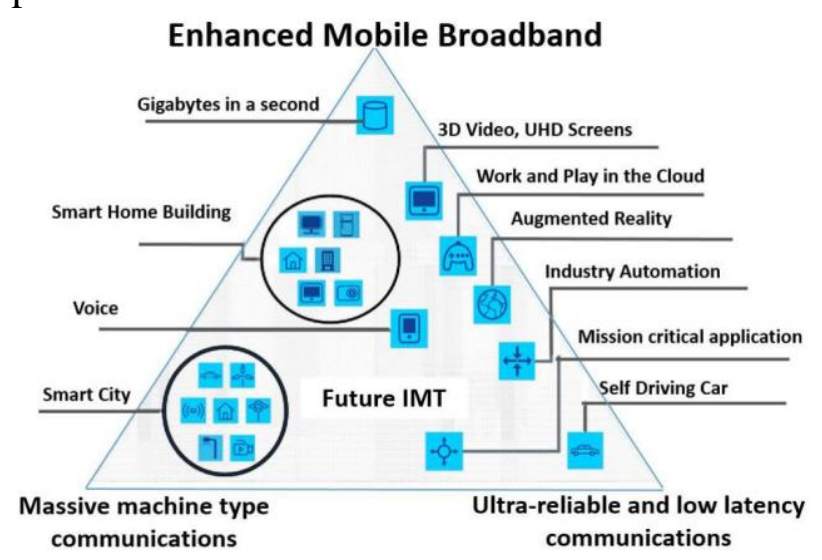

Figure 3: Usage scenarios for $5 \mathrm{G}$, as defined by ITU in IMT2020 document

1. Enhanced Mobile Broadband: This caters to future requirements of mobile broadband users who need higher bandwidth for personal use. Key driver for bandwidth demand will be videos and some high bandwidth consumer applications.

2. Ultra-Reliable and Low Latency Communication(URLLC): This caters to needs of industries where speed and reliability are of extreme importance.

3. Massive Machine Type Communications: This caters to M2M/IoT requirements which have high density of connected sensors/devices/machines, etc. which will form Smart City eco-system in near future.

The three usage scenarios have been plotted on extremes of triangle. These represent application services which have very specific network requirements (e.g. high bandwidth, low latency, connection density etc.). The other services mentioned in triangle have varying degrees of overlap in their network requirements (e.g. medium bandwidth and medium latency). In this way, all possible usage scenarios for $5 \mathrm{G}$ have been envisioned to fall under (either exclusively or as combination to various degrees), the 3 key usage scenarios as mentioned previously.

In order to fulfill requirements for key usage scenarios, following objectives have been identified for $5 \mathrm{G}[3]$.

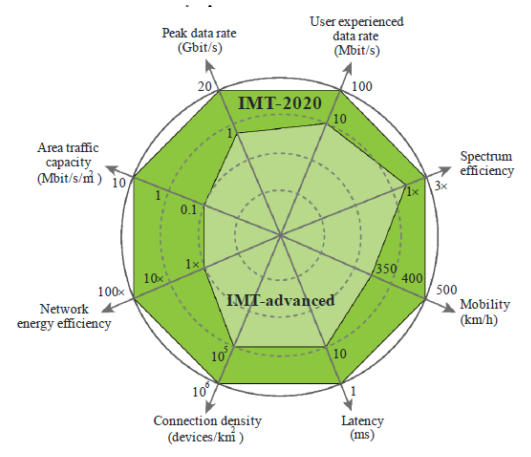

Figure 4: Key objectives that 5G(IMT2020) network needs to fulfil vs 4G(IMT advanced)

ITU[4] defines these network parameters as followings: 
- Peak data rate: Maximum achievable data rate under ideal conditions per user/device (in Gbit/s).

- User experienced data rate: Achievable data rate that is available ubiquitously across the coverage area to a mobile user/device (in Mbit/s or Gbit/s).

- Latency: The contribution by the radio network to the time from when the source sends a packet to when the destination receives it (in $\mathrm{ms}$ ).

- Mobility: Maximum speed at which a defined QoS and seamless transfer between radio nodes which may belong to different layers and/or radio access technologies (multilayer/-RAT) can be achieved (in $\mathrm{km} / \mathrm{h}$ ).

- Connection density: Total number of connected and/or accessible devices per unit area (per km2).

- Energy efficiency: Energy efficiency has two aspects:

$\circ$ on the network side, energy efficiency refers to the quantity of information bits transmitted to/ received from users, per unit of energy consumption of the radio access network (RAN) (in bit/Joule);

$\circ$ on the device side, energy efficiency refers to quantity of information bits per unit of energy consumption of the communication module (in bit/Joule).

- Spectrum efficiency: Average data throughput per unit of spectrum resource and per cell (bit/s/Hz).

- Area traffic capacity: Total traffic throughput served per geographic area (in Mbit/s/m2).

For people familiar with networks, looking at previous two diagrams should raise question on how can so many different requirements be met by single network. Given the fact that $5 \mathrm{G}$ will be able to do this, should help us realize the complexities involved. Based on usage scenarios, we can envision three key types of networks needed. The three networks correspond to key usage scenarios that we had discussed in previous section.

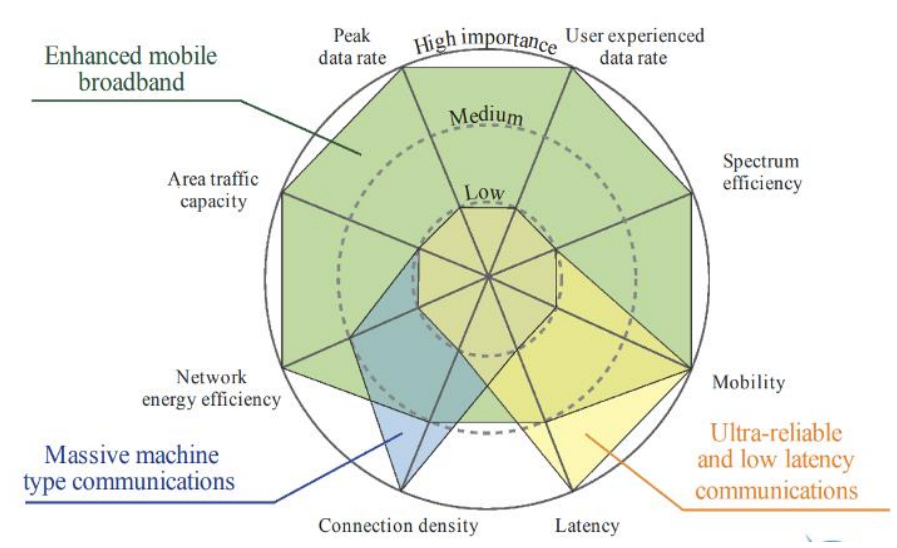

Figure 5: Three types of networks that are envisioned within $5 G$. Source: IMT

So, for Enhance Broadband, designed network should have high Peak Data rate, Spectrum efficiency and high traffic carrying capacity as they all deal with large amount of data that can be uploaded/downloaded through network. But, in case of Ultra-reliable and Low Latency Communication, Mobility and Latency are of most importance and high bandwidth is not needed. For Massive machine type communications, connection density is most important parameter. Thus, all three types of networks would be created with different characteristics, but all will be able to interwork and co-exist under bigger umbrella of $5 \mathrm{G}$.

\section{Key technologies}

To achieve vision of 5G, following are two key areas where ground breaking technology innovations are happening:

- mmWave radio systems and architecture

- Using Cloud for Telecom network

mmWaves are expected to provide high bandwidth required in $5 \mathrm{G}$ and Cloud deployments to help create flexible network architecture which enable various $5 \mathrm{G}$ use cases. Let us look at these technologies in detail.

\section{1 mmWave radio systems and architecture}

Shannon's theorem [5] defined achievable channel capacity as function of channel bandwidth(B),

$$
C=B * \log 2(1+S / N)
$$

signal $(\mathrm{S})$ and noise $(\mathrm{N})$ power. The formula is shown below.

It is evident that channel capacity is directly proportional to bandwidth. Higher the bandwidth, higher would be channel capacity. Across various 
generations of mobile networks, spectral efficiency and cell size have been optimized to get maximum capacity possible. In today's mobile communication network, we have been using frequencies till about $3 \mathrm{Ghz}$, and there is no potential to get additional BW in sub $3 \mathrm{GHz}$ range. So, only option possible is to move towards higher frequencies in spectrum where more bandwidth is available.

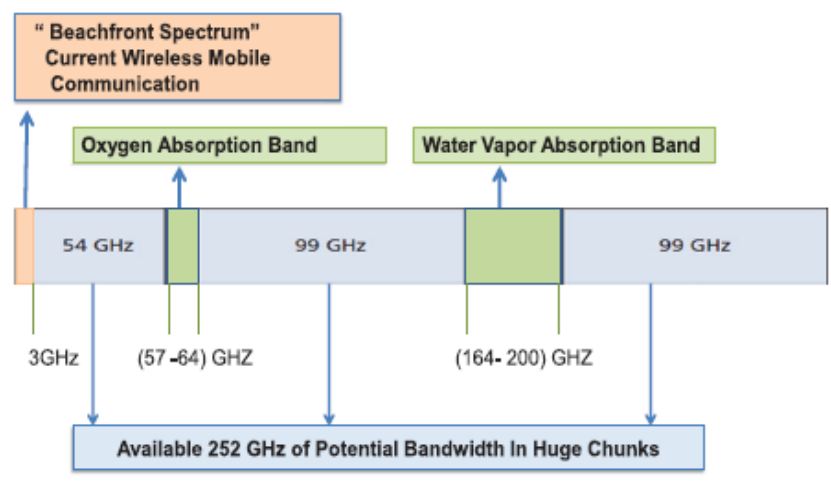

Figure 6: Spectrum availability and usage[8]

It has been observed that in mmWave range(30$300 \mathrm{Ghz}$ ), huge chunks of frequency bands are available for use. It was determined that even if small fraction of available bands was made available for mobile communication, the capacity of data transmission would increase tremendously. Also, it is a known fact that higher frequencies require

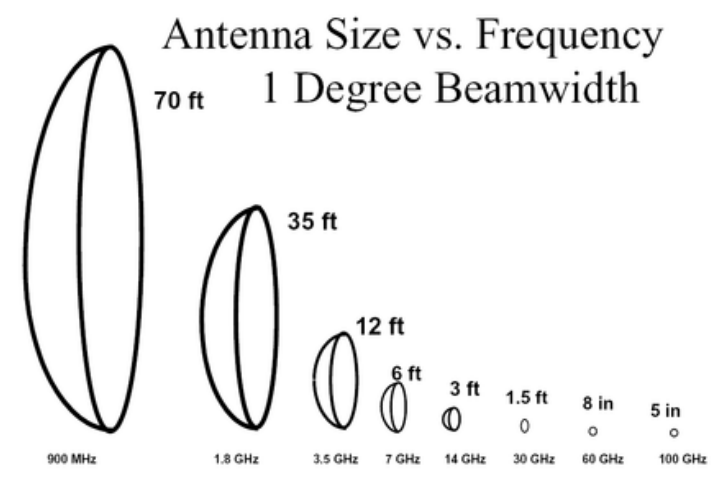

smaller antenna sizes[7]. This is also a big advantage as it would be possible to reduce antenna sizes to fit even smaller machines/devices.

Figure 7: Antenna size required at various frequencies. Source Lightpointe

Following are some of frequency bands being considered globally for 5G networks. WRC19(Agenda item 1.13) [18] which takes place in 2019, will help standardize bands, which is an essential ask for global deployment and reach economies of scale.

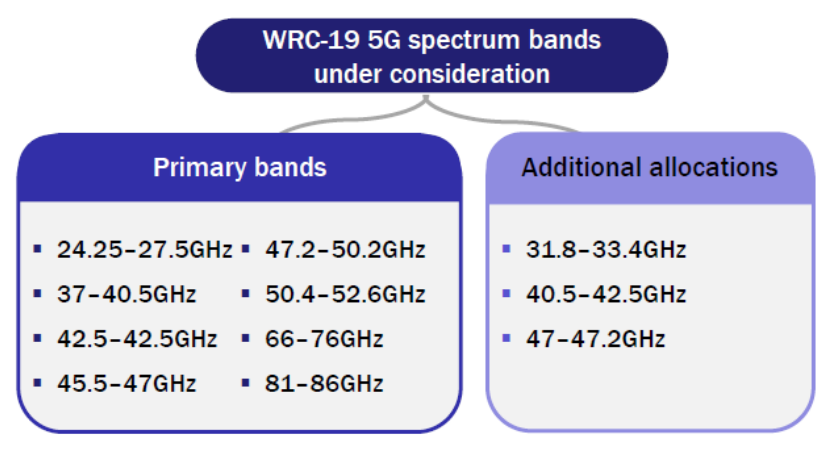

Figure 8: Source Analysys Mason

In India, 3 bands are expected to be dedicated for 5G:

- 24.25 to $27.5 \mathrm{GHz}$

- 37 to $40 \mathrm{GHz}$

- 31.8 to $33.4 \mathrm{GHz}$

Before we look at $\mathrm{mmW}$ use cases, let us look at radio technologies which will be used extensively in

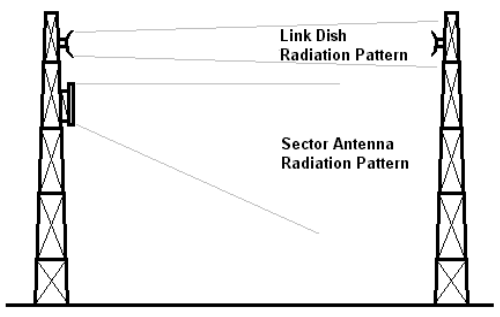

combination with $\mathrm{mmW}$ to achieve high data rates. These technologies are "beam forming" and MIMO (Multiple Input Multiple Output)[9].

A typical antenna has a very wide beam, could be around $120 \mathrm{deg}$. Using array of antenna elements, it is possible to transmit highly concentrated beam instead of beam that has wide arc. The concentrated beams make it possible to send multiple steams using same antenna and medium, thus multiplying the amount of data being transmitted by antenna. This is called bean forming and is being used extensively today.

Figure 9: Propagation of waves from Antenna

A simple way to explain beam forming is to assume a person sitting on antenna pole with a torch. The beam of torch spreads in a huge arc and energy gets dissipated across a large area. In same way, when traditional antenna wants to transmit data towards receiver, it transmits data in huge arc, and receiver receives a very small fraction of overall power transmitted. 
Now, assume that the person is sitting on pole with laser pointer. The light of laser is concentrated in very small area and also reaches a long distance. It is now possible for him to send flashes of light to multiple people in short duration of time. In fact, he can also use multiple lasers to send out parallel beams. As laser light does not spread, each laser beam is independent of others. Thus, in place of having just one torch transmitting information, we can have multiple laser beams transmitting data in parallel without interfering with each other, thus increasing available bandwidth and power of signal that is received by receiver.

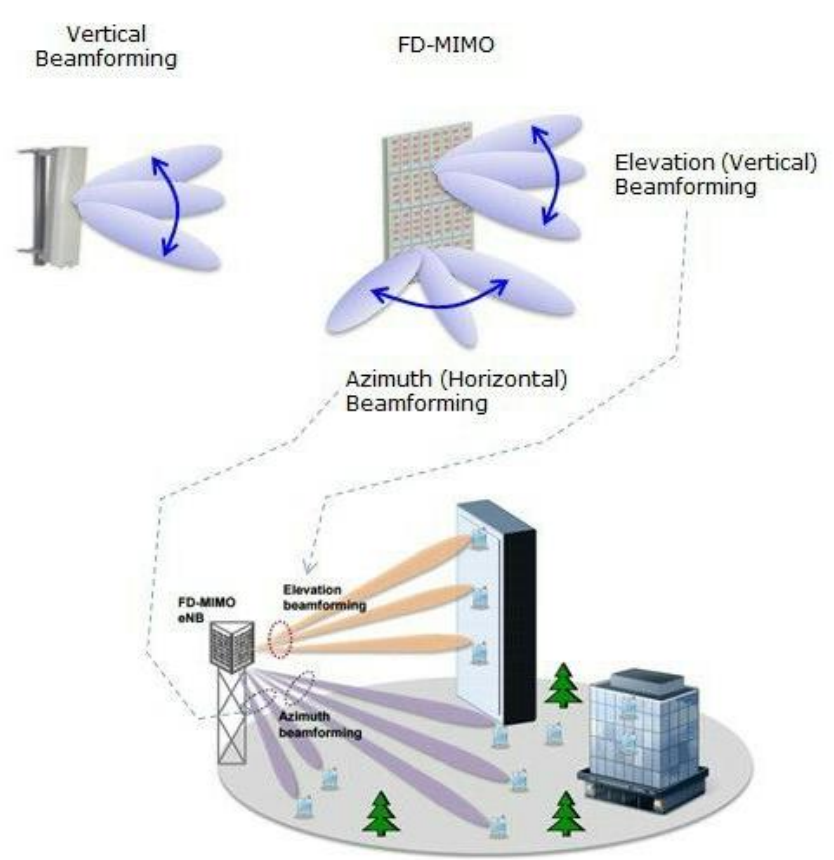

Figure 10: Beam forming

MIMO is the ability to send and receive multiple streams of data using same antenna array. This is done by using large number of antennas per base station. The concept of superposition of waveforms is used in MIMO to create narrow beams. In below example, we can see that the 2 receivers receive data from 4 transmitters through 4 isolated radio paths. So, each receiver is able to receive three times more data than it would if there was only 1 transmitter. This shows how capacity is enhanced using multiple transmit receive streams.

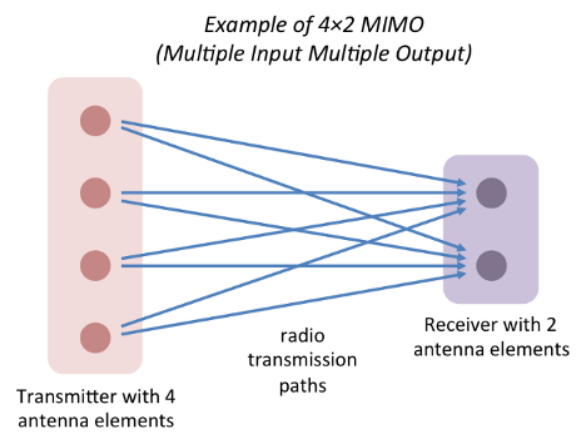

Figure 11: Concept of Multiple In Multiple Out

\section{mmW use cases:}

Till now we have learnt that mmWave have very high frequencies and can provide high bandwidth compared to frequencies used in today's Mobile Communication. However, what is also important is to note that higher frequencies lead to reduction in propagation distance as they are not able to penetrate obstacles. What this means is that mmWave will propagate much less than waves with lower frequencies. So, use of $\mathrm{mmW}$ is possible across short distances only.

Keeping this in mind, mmW's are planned to be used in following ways for $5 \mathrm{G}$ :

For creating small cells: Small cells are radio cells which have low power and cover very small area and are generally used to enhance capacity. A Macro cell provides coverage to wide area. But, due to huge area covered (and also due to serving large number of subscribers), macro cell may not be able to provide high data rates. It has also been seen that, high capacities are required is small pockets which have high density of users i.e. bus stops, stations, office buildings, malls, etc. It is for such scenarios that small cells are being deployed. Even in 5G, concept of small cell remains same. What changes is the frequency deployed on small cells. In $5 \mathrm{G}$, it is expected that $\mathrm{GHz}$ range $(5 \mathrm{GHz}+)$ would be used in small cells which will be able to provide Gbps download speeds.

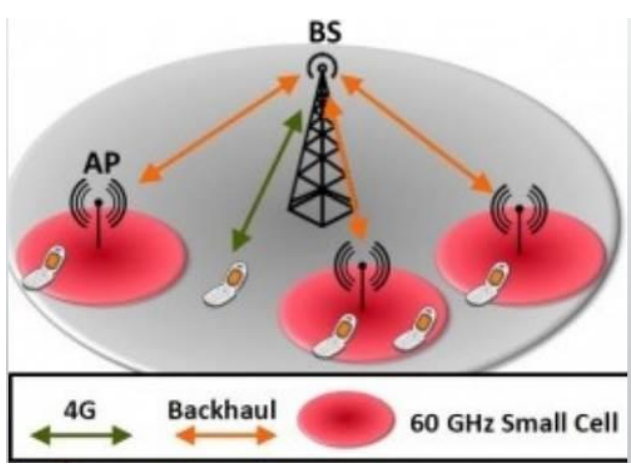

Figure 12: Deployment of Small cells in 5G

Backhaul \& Fronthaul: The other way in which $\mathrm{mmW}$ can be deployed is as backhaul and front haul medium. This is because mmWave provide very high bandwidth and narrow beam width, thus making mmWave a viable fiber replacement 
technology. Additionally, with narrow beam width, interference reduces and highly directional beams will lead to greater frequency reuse and improved security.

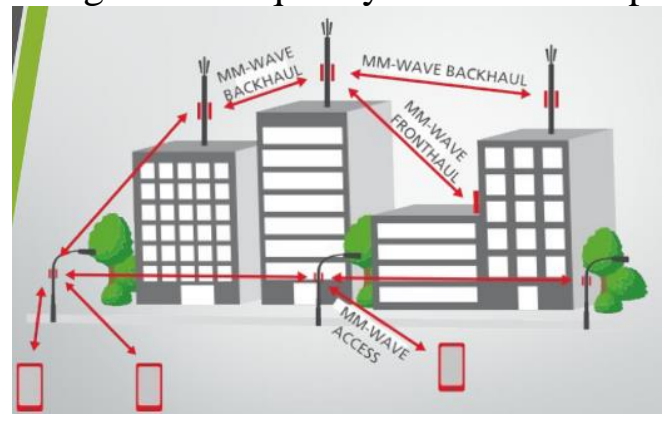

Figure 13: Concept of front and back haul using mmWave

By using mmW back and front haul, operators would have a lot more flexibility in planning their networks as dependencies on fiber would no longer exist. The ability to set up $\mathrm{mmW}$ high bandwidth connection quickly and at low costs, will accelerate network deployment process and also provide potential financial benefits visa vi fiber deployment for front and back haul[8].

\subsection{Network on Cloud}

The concept of cloud has matured a lot from ARPANET days, when it was used as means to connect few computers, to today's world where Cloud Computing is becoming de-facto standard. Cloud computing enables virtualization of physical resources (compute, storage, network) so that the application is isolated to underlying physical infrastructure. This isolation provides many benefits, mainly, resource pooling, elasticity, and on-demand availability.

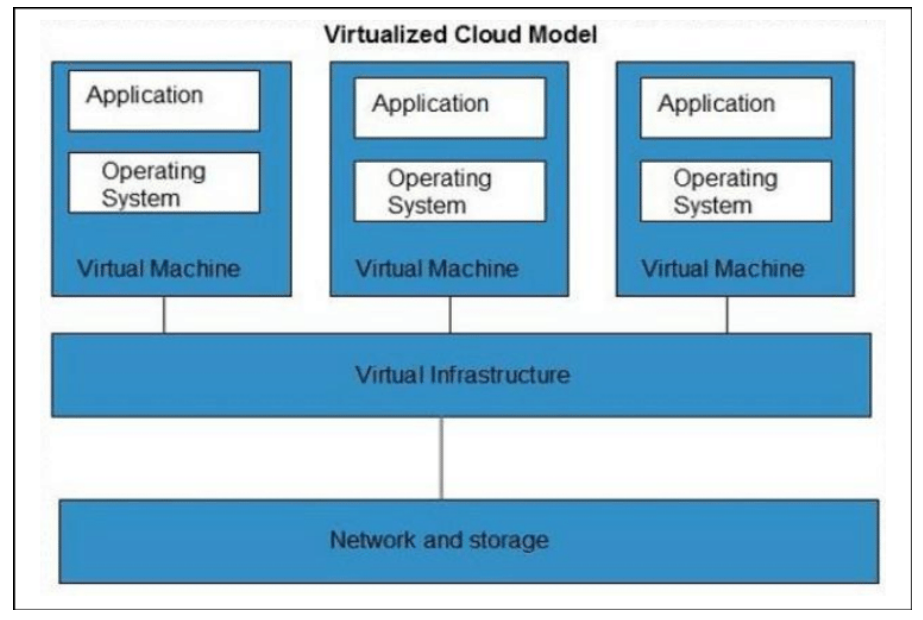

Figure 14: Cloud framework

Almost all major operators and vendors have been experimenting with cloud, either for IT services(OSS/BSS) and more recently for Core \& RAN services [11]. With 5G, Cloud becomes an integral part of Telecom network. This is because a lot of what $5 \mathrm{G}$ would like to achieve, is not possible using traditional hardware software model used in Telecom industry.

This paper focuses on 3 key concepts which will be used in 5G:

- Network Virtualization

- Edge computing

- Network Slicing

\subsubsection{Network Virtualization:}

Network virtualization is exactly same as concept of

\section{TRADITIONAL AND VIRTUAL ARCHITECTURE}

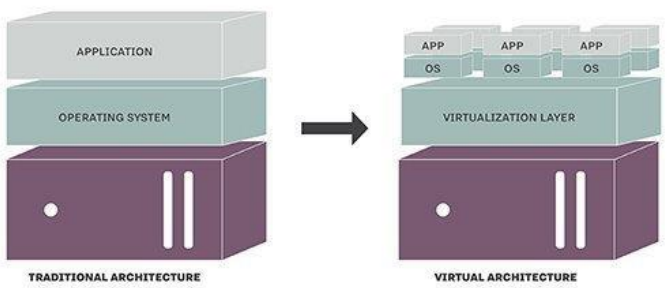

Virtualization used in IT systems. It decouples the hardware from the software, which provides an ability to deploy software without dependencies on underlying layers.

Figure 15: Hardware architecture

ETSI had established a specification group in 2012, which is defining specifications for Telco cloud. Given below is snapshot of ETSI's cloud architecture and specifications associated with it. 


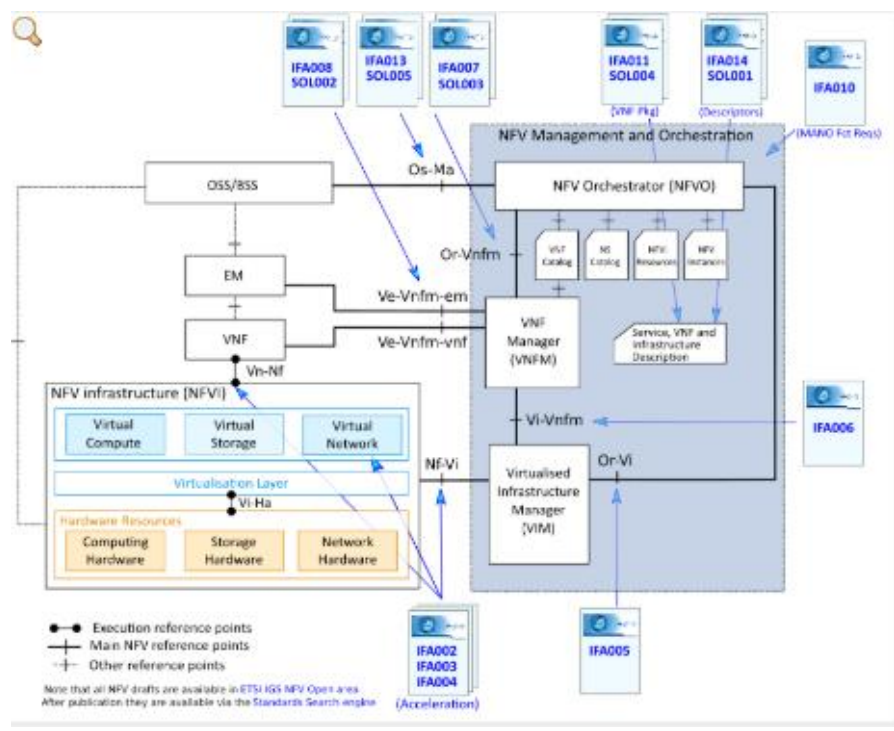

Figure 16: ETSI's work on Standards

ETSI specifies following advantages of virtualized network:

- Decoupling software from hardware: Enables independent evolution of both hardware and software components

- Flexible network function deployment: Ability to deploy/instantiate network services automatically over virtualized infrastructure

- Dynamic operation: Ability to increase or decrease deployments based on traffic/demand

Simplified view of ETSI's NFV framework is shown below. It has three main working domains:

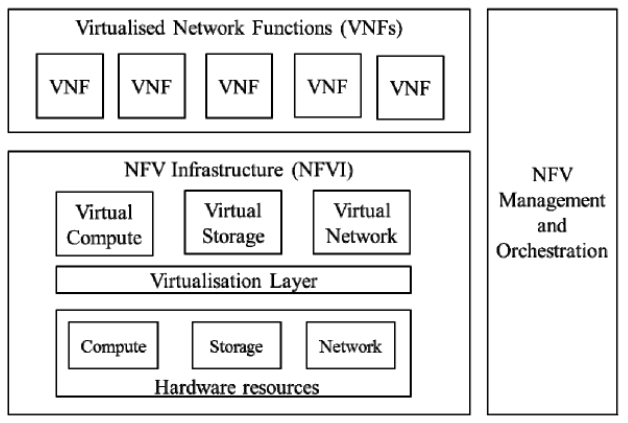

Figure 17: Hardware architecture

1. Virtual Network Functions $(N F V)$ : is software implementation of network function (e.g. packet gateway, etc.) capable of running over NFVi

2. NFV Infrastructure(NFVi): includes diverse range of physical resources which are virtualized and available for NFV

3. NFV Management \& Orchestration(MANO): takes care of management and orchestration of physical and virtual resource along with life cycle management of NFV's

4. Additionally, there is also an OSS/BSS function

Shown below is example of how NFV is deployed on Telco cloud. This is deployment of vEPC and vIMS is done by Vmware and Ooredoo[12].
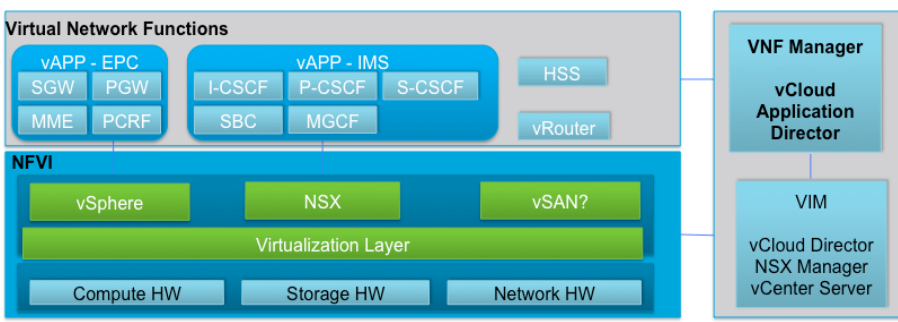

Figure 18: Sample deployment of Virtualized Network applications on Telco Cloud

As is evident, the service functions are now pieces of software(VNF) running on virtualized infrastructure(NFVi). VNF Manager and VIM manage the various hardware and software elements of solution along with lifecycle management.

Explained below are some of typical telecom scenarios and how they will change on cloud:

Installation of new service: In traditional ecosystem, the hardware and software would be procured. Hardware would be installed, on which software would be installed, configured and deployed. This is long and time consuming process.

Instead, on telco cloud, following would be key steps which can be completed in matter of minutes:

- Need for new service is identified

- Trigger given to VNF Manager and VIM

- Based on identified infra needs, VIM provides computing, storage and networking resources

- VNF manager then creates instance of $\mathrm{VNF}$ (service) using provided virtual resources and configurations

Note: VNF Manager should have the required configuration files and data related to new VNF in its database. Only then can it instantiate and VNF automatically.

Expansion of computing resources: In traditional environment, when need for additional processing is identified, Computing card/hardware is ordered. This is then installed on the platform and made live. 
These activities would also need to be done during off-peak hours to avoid any service outage.

On Telco cloud, this would be done as follows:

- OSS/BSS detects high virtual CPU utilization of VNF and creates alert

- Information is passed to VNF Manager which requests for additional virtual processing resources

- VIM aligns and provides additional resources to $\mathrm{VNF}$

- OSS/BSS detects reduction in virtual CPU utilization and clears its alert

Both these scenarios are possible as hardware and software have been de-coupled. The platform is able to provide required virtual hardware resources as required by service instance and in same way, the services are not concerned with status of physical hardware. This makes managing both hardware and software simple and non-service impacting. This makes it possible to run isolated virtual networks on same hardware.

\section{Edge Computing}

Traditionally, computing resources have always been deployed centrally. The deployment could be Centralized for Country, City or smaller geographical area. This provided the means to better manage computing infra and control costs.

With drop in cost of compute and expansion in high bandwidth, low latency applications, edge computing has gained prominence. Wikipedia defines edge computing as "pushing the frontier of computing applications, data, and services away from centralized nodes to the logical extremes of a network".[13]. It enables analytics and data gathering to occur at the source of the data. "

We have seen in initial sections of this paper that $5 \mathrm{G}$ aims to support Ultra-reliable and Low latency communication services. To be able to achieve this, it is important to have computing resources very close to the devices/equipment. This is because applications like remote surgery, AR/VR, etc. cannot tolerate delays even in scale of 10's of milliseconds. Another important aspect is the high volume of data being sent from devices to servers from services such as video surveillance/analytics, AR/VR, etc that have recently come up.

Edge computing overcomes the issue of transportation delay and overload of backhaul network by having application servers very close to devices. Though the concept of edge devices has been implemented in $4 \mathrm{G}$ networks, in $5 \mathrm{G}$, it is completely integrated to provide superior quality and performance of experience [14].

ITU has coined term "Multi Access Edge Computing(MEC)" for edge computing support on mobile networks. Due to deep coupling of $5 \mathrm{G}$ network MEC, it is possible for network and MEC to collaborate, interact in traffic routing and policy control functions, thus creating a powerful environment on which services can be run.

ETSI[15] defines 4 possible deployment models for MEC where UPF is User plane function.

1. Collocated with base station

2. Colocated with transmission node

3. Colocated with network aggregation point

4. Colocated with core network functions
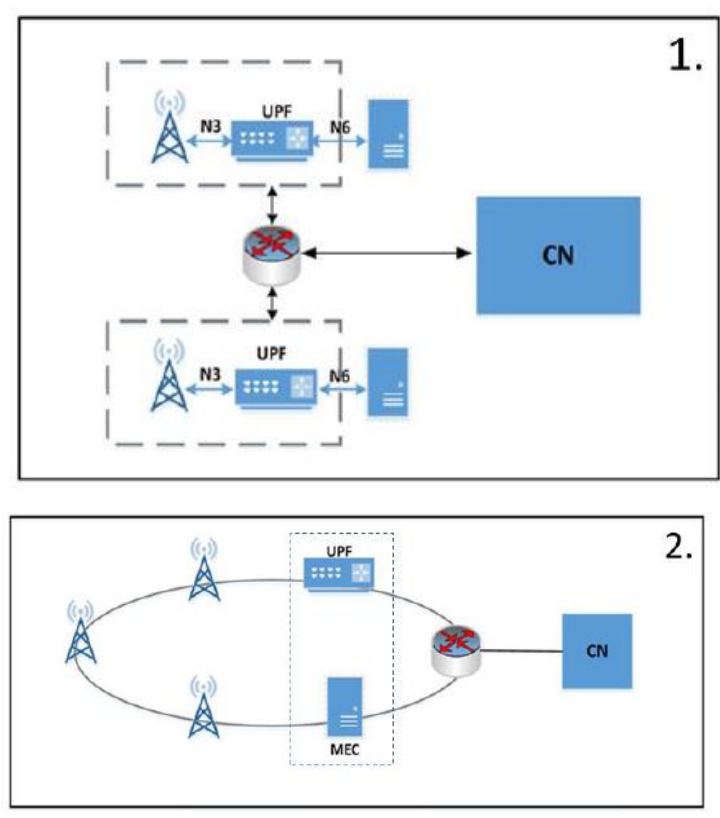

2.

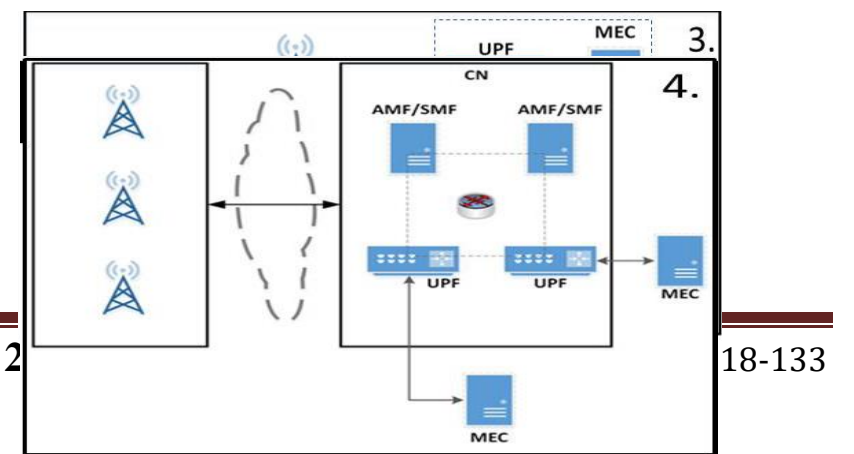


Figure 19: MEC deployment models [15] In another paper available on ETSI's portal, some use cases for MEC have also been described. Given below are 2 of the many use cases that are explained:

MEC for Video analytics:

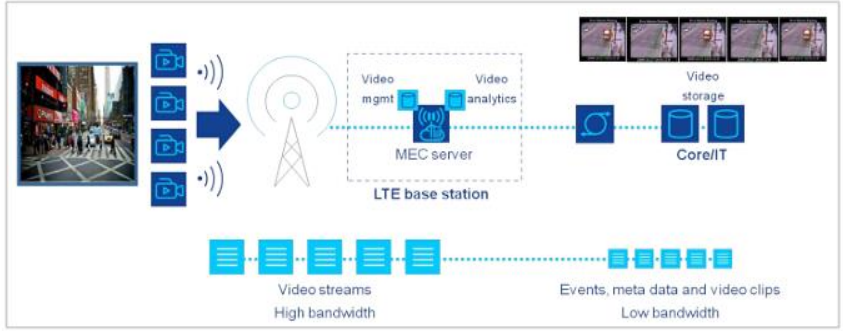

Figure 20: Deployment of MEC for Video analytics In this scenario, MEC server capable of performing Video analytics is placed at base station. The feed coming from the cameras are analyzed in MEC server and only the relevant portion of feed or triggers are then sent to Application servers residing in Core Data centers. Due to MEC server, the complete video is not sent back to core data center thus saving substantial bandwidth and also increasing responsiveness as data is processed near devices.

\section{$A R / V R$ content delivery.}
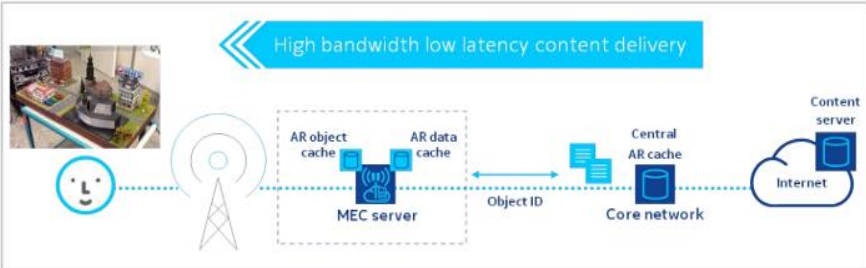

Figure 21: Deployment of MEC for AR/VR

In this, the AR content is cached on MEC servers at base station. As content is available on edge, the relevant data can be sent to AR device without involving the Application servers at Core data center, thus enhancing user perception of service and avoiding load on back haul network.

Besides such application, MEC will have infinite potential in IoT \& Industry 4.0 type of vertical applications due to physical proximity of service which reduces response time.

\section{Network Slicing}

Mobile networks have been primarily built for consumers using mobile devices. The evolution has been from all voice, to simple data ,to current networks which provide high speed data. Another major breakthrough has been all IP network which was introduced as $4^{\text {th }}$ generation network or $4 \mathrm{G}$. In spite of great strides made in this, the basic architecture of network has not changed a lot. The mobile network mainly consists of hardware and software for:

- Core Network where all the network functions reside

- Transmission network which connects core to RAN

- Radio Access Network which connects users to mobile network

These basic elements have remained the same and so has architecture and all other advantages and disadvantages associated with this architecture.

\section{Need for Network Slicing}

The vision of $5 \mathrm{G}$ is to build an integrated network which could be used for consumers and for various types of enterprises also.

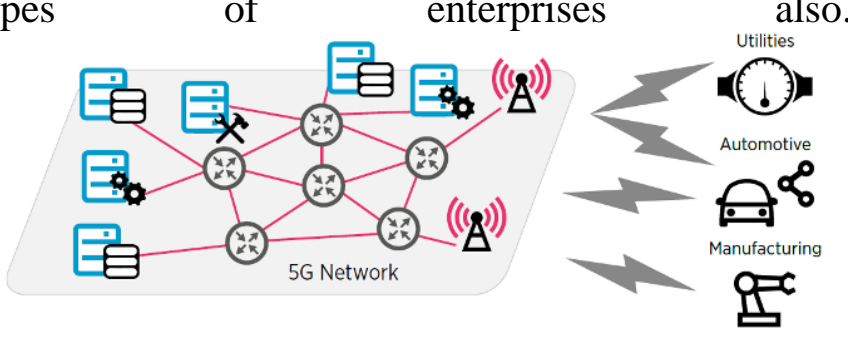

Figure 22: Flat 5G network[16]

For consumers, the key network requirement is speed/bandwidth, whereas enterprises can have diverse requirements such as low latency, very high uptimes, high reliability, huge volumes of connections etc. The typical architecture used in $4 \mathrm{G}$ cannot satisfy all these requirements. $5 \mathrm{G}$ is designed to support all these requirements using latest IT technology enhancements.

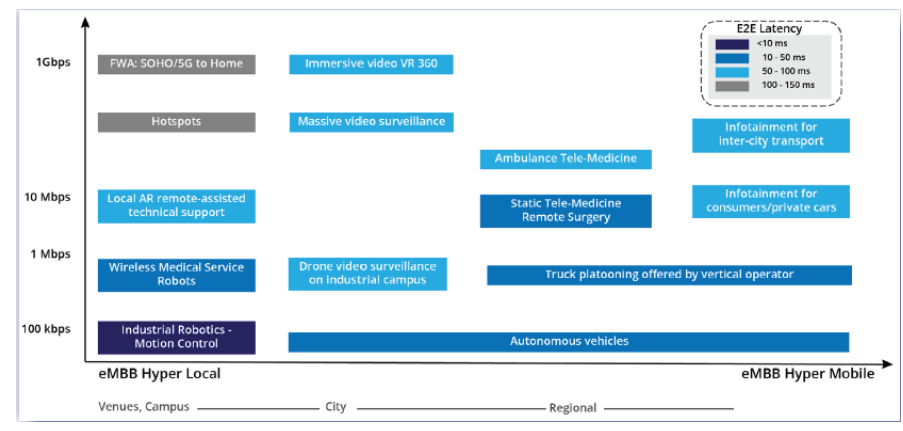

Figure 23: GSA's view on service requirements by verticals

With advent of Virtualization and Cloud technology, the concept of shared resources has become 
common. The ability to use same physical resources and run virtual applications/services transformed the IT industry. On same lines, Cloud and Virtualization are being used to create next generation of Mobile Networks. These concepts have been integrated within $5 \mathrm{G}$ specifications and have given rise to concept of "Network Slicing".

\section{What is network slicing}

The concept of Network slice is creation of user defined virtual network/networks over an existing physical network. A report in GSMA[16] defined it as follows:

\section{From a mobile operator's point of view, a network slice is an independent end-to-end logical network that runs on a shared physical infrastructure, capable of providing a negotiated service quality. The technology enabling network slicing is transparent to business customers. \\ A network slice could span across multiple parts of the network (e.g. terminal, access network, core network and transport network) and could also be deployed across multiple operators. A network slice comprises dedicated and/or shared resources, e.g. in terms of processing power, storage, and bandwidth and has isolation from the other network slices.}

With ability to create virtual slices over same physical network, it becomes possible to create separate virtual networks suited for specific purposes. The below figure tries to explain concept of slice for verticals.

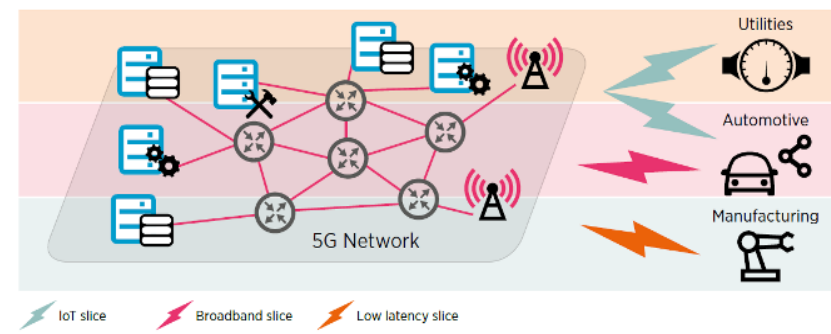

Figure 24: Indicative view of network slices[16]

The sample physical network shown above is divided into 3 slices. The slice for IoTs will be configured to support high connection density and bursty transmissions. Whereas, Broadband slice will be created in such a way that it supports high bandwidth and mobility. As name suggests, the low latency slice will have very low round trip time for data packets and also have very high reliability. As per need of industry/application/service, access could be provided to the required slices. E.g. for Automotive, access can be provided to broadband slice for in-car entertainment service and IoT slice for capturing car performance and troubleshooting data.

A slice could be for any part of network. In image shown below, S1 is slice at Radio network only. Rest of the network is common to all slices. Similarly, Slice S5 is a dedicated e2e slice which does not share any of its virtual resources with other slices. What is also evident is that Slice S1 has least isolation, but will be most cost efficient slice. So, this could be used by MVNO's to provide isolated RAN network work for their subscribers, while other parts of network remain common. Whereas Slice S5 has maximum isolation and will also incur most costs. This may be used by URLLC networks (autonomous vehicles) as it will act as separate isolated network whose characteristics could be tweaked for high reliability and low latency. Thus, the slice will have to be created keeping the service requirements in mind with various combinations of slices possible in network.

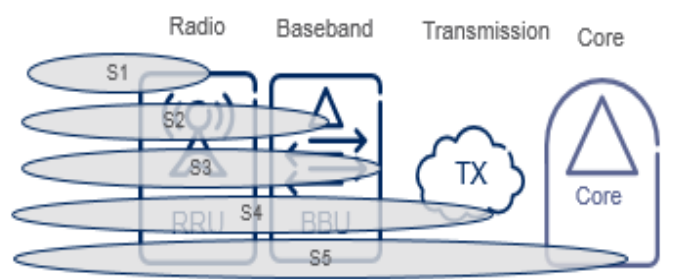

Figure 25: Possible ways to create slices

The concept of network slicing is being tested by vendors and operators, however, this is still a few years from commercial deployments.

\section{Conclusion}

Mobile communication has been one of the key digital enablers directly impacting countries GDP. All previous generations of wireless technologies have helped economy grow in their own ways. $5 \mathrm{G}$ is also expected to do the same.

Two most important changes that we would see in $5 \mathrm{G}$ network is use of mmWave and Network Slicing. mmWave will enable Gbps rates within wireless environment, whereas network slice will help create virtual networks over single physical network, thus enabling the creation of virtual networks specifically designed for application/users requirements.

5G poses a very unique challenge to telecom industry. Besides technology challenges, deep integration with vertical industries is something 
which has never been done by Telecom industry. Besides this, huge investment and unclear business models also makes adoption of $5 \mathrm{G}$ a very risky business decision.

Given the fact that standards are still being developed, full-fledged commercial deployments are still quite a few years ahead. However, the importance given to $5 \mathrm{G}$ by almost all Govt. bodies across the world makes me believe that this will be a game changer in wireless world. With China and America leading the $5 \mathrm{G}$ roll out competition, it is just a matter of time before world takes next big leap and moves to $5 \mathrm{G}$.

\section{References}

[1] Internet of things forecast, Ericsson. Available: https://www.ericsson.com/en/mobilityreport/internet-of-thing

[2] How 5G will change the world, World Economic Forum. Available: https://www.weforum.org/agenda/2018/01/theworld-is-about-to-become-even-moreinterconnected-here-s-how/

[3] Minimum requirements related to technical performance for IMT-2020 radio interface(s), ITU Publications

[4] IMT Vision - Framework and overall objectives of the future development of IMT for 2020 and beyond. ITU Publications.

[5] Shannon's theorem. Available: http://www.inf.fu-berlin.de/lehre/WS01/19548U/shannon.html

[6]LTE Bands. http://niviuk.free.fr/lte band.php

[7] $60 \mathrm{GHz}$ Technology Overview. Available: https://www.lightpointe.com/60-ghztechnology-overview.html

[8] Next Generation Wireless: Riding on back of millimeter waves. Available: https://www.wirelessweek.com/article/2013/12 /next-generation-wireless-riding-backmillimeter-waves

[9] MIMI, Wikipedia. Available: https://en.wikipedia.org/wiki/MIMO

[10]Cloud storage, Wikipedia. Available: https://en.wikipedia.org/wiki/Cloud_storage\#ci te note-origin-1

[11] Telefónica's UNICA architecture strategy for network virtualization. Available: https://www.telefonica.com/documents/23283/
140082485/Telefonica_Virtualisation_gCTO

FINAL.PDF/bb870423-cc0f-409f-6638-

53af4cf2b24b?version $=1.0$

[12] VMware Telco NFV Blog, Available: https://blogs.vmware.com/telco/tag/ooredoo/

[13]Edge computing, Wikipedia. Available: https://en.wikipedia.org/wiki/Edge_computing

[14] MEC in 5G, ETSI. Available: https://www.etsi.org/images/files/ETSIWhiteP apers/etsi_wp28_mec_in_5G_FINAL.pdf

[15] MEC in 5G networks, ETSI White paper No.28

[16] Introduction to Network Slicing. Available: https://www.gsma.com/futurenetworks/wpcontent/uploads/2017/11/GSMA-AnIntroduction-to-Network-Slicing.pdf

[17] Mobile Traffic Analysis by Application, Ericsson. Available:

https://www.ericsson.com/en/mobilityreport/reports/june-2018/mobile-trafficanalysis-by-application

[18] WRC-19 Agenda. Available: https://www.itu.int/en/ITU-R/studygroups/rcpm/Pages/wrc-19-studies.aspx

\section{Author Profile}

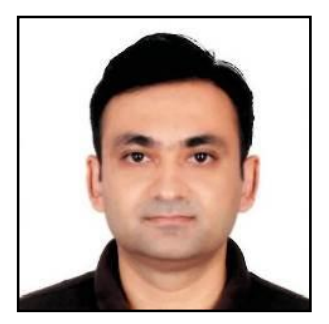

Rahul is a Senior Consultant working with Ericsson Global Services India Ltd. He has 15+ years of

Industry experiences and is currently involved with Business and Operations consulting projects. He has keen interest in understanding new technologies and their application in today's Business environment. Rahul has an Engineering degree with specialization in Telecommunication from Mumbai University. 